\title{
Opioid Use Disorder in Pregnancy: Leveraging Provider Perceptions to Inform Medication-Assisted Treatment
}

Doris Titus-Glover ( $\nabla$ dtitus-glover@umaryland.edu )

University of Maryland, Baltimore https://orcid.org/0000-0002-1812-8868

Fadia T Shaya

University of Maryland School of Pharmacy

Christopher Welsh

University of Maryland School of Medicine

Danya M Qato

University of Maryland School of Pharmacy

Savyasachi Shah

University of Maryland, School of Pharmacy

Laura E Gressler

University of Maryland, School of Pharmacy

Rebecca Vivrette

University of Maryland School of Medicine

\section{Research article}

Keywords: Qualitative pilot study, medication-assisted treatment, opioid use disorder, pregnant women, pregnancy and, healthcare provider

Posted Date: May 5th, 2020

DOI: https://doi.org/10.21203/rs.3.rs-24823/v1

License: (a) (1) This work is licensed under a Creative Commons Attribution 4.0 International License. Read Full License

Version of Record: A version of this preprint was published on March 10th, 2021. See the published version at https://doi.org/10.1186/s12913-021-06182-0. 


\section{Abstract}

Background: Medication-assisted treatment is recommended for pregnant women with opioid use disorder (OUD), yet only $50 \%$ receive the treatment. Knowledge deficit, limited access to prescribing providers, and complex delivery models are known barriers to treatment. Strategies for improvement of medication-assisted treatment during pregnancy require multi-disciplinary provider input. The objective of this study is to explore provider perceptions and factors critical to medication-assisted treatment delivery for pregnant women with OUD.

Methods: A qualitative research approach was used to gather data from individual provider and group semi-structured interviews. Providers $(n=12)$ responded to questions in several domains related to perceptions of medication-assisted treatment, treatment delivery, access to resources, and challenges/barriers. Data were collected, transcribed, coded (by consensus) and emerging themes were analyzed using grounded theory methodology.

Results: Emerging themes in this pilot study, revealed persistent provider knowledge/training gaps, inadequate service coordination, inconsistent treatment guidelines, stigmatization, and lack of resources such as housing and transportation. Providers found medication-assisted treatment offered pregnant women with OUD, a "lifeline."

Conclusions: In this pilot study, we conclude that, MAT variances can be improved by leveraging healthcare provider perceptions, direct experiences and recommendations towards a team-based, patientcentered integrated approach.

\section{Introduction}

Maternal use of prescription opioids and illicit drugs increased from 1.5 to 6.5 per 1000 deliveries from 2000 to 2014, according to the Centers for Disease Control and Prevention. ${ }^{1}$ Under-treatment of pain and provider prescribing practices were contributory factors associated with this increase. ${ }^{2,3}$ The incidence of pregnant women with opioid use histories presenting to addiction treatment centers also increased from $2 \%$ to $28 \%$ between 1992 and $2012 .{ }^{4}$ Opioid use disorder (OUD) ${ }^{5}$ predisposes pregnant women to adverse birth and maternal/neonatal outcomes. ${ }^{6,7}$ Recent data from state maternal mortality reviews identified substance use/overdose deaths as a major cause of death in pregnant women. ${ }^{8,9}$ Among opioid-exposed babies, national incidence rates for neonatal abstinence syndrome have increased by at least five times since 2000 , concurrent with the rise in OUD. ${ }^{10}$

The recommended treatment for pregnant women with OUD is medication-assisted treatment (MAT). 6,11,12 MAT consists of opioid agonist pharmacological treatment options, such as buprenorphine (Subutex ${ }^{\circledR}$ and Suboxone ${ }^{\circledR}$ ) or methadone. ${ }^{13-15}$ Both medications have been proven to be effective in reducing severe withdrawal symptoms and improving adherence to treatment when combined with behavioral counseling and prenatal care. ${ }^{16}$ Buprenorphine, a partial agonist with unique pharmacological 
properties has advantages over methadone, a full agonist, which can lead to improved treatment compliance. ${ }^{15}$ Despite these promising outcomes, only $50 \%$ of pregnant women on Medicaid, diagnosed with OUD received MAT. ${ }^{17}$ This less than optimal utilization has been mostly attributed to provider, health systems, and patient factors. 6,10

The Substance Abuse and Mental Health Services Administration (SAMHSA), among several leading agencies, encouraged obstetricians and other providers to play "an active role" in screening, identifying, treating, and referring patients appropriately, along with adapting strategies for multidisciplinary coordination to address deficiencies in treatment. ${ }^{18}$ Under the Drug Abuse Treatment Act (DATA) of 2000, SAHMSA created a waiver program that certifies qualifying providers to prescribe buprenorphine. ${ }^{19}$ This critical pathway to increase treatment and access to MAT was emphasized in the final report of the President's Commission on Combating Drug Addiction and the Opioid Crisis. ${ }^{20}$

Among patient factors, the challenges in managing pregnant women with OUD are compounded by mental health co-morbidities ${ }^{21}$ often outside the scope of any one healthcare provider's expertise. While most studies focused on the pharmacological benefits of MAT, the challenges of treatment for providers have not been explored to the best of our knowledge. The study's objective was therefore to understand factors critical to delivering the recommended treatment among pregnant women with OUD from the provider perspective, given that pregnancy is a time of multiple patient-provider interactions.

The lead author has built a career in maternal and child health and initiated this study during postdoctoral training in behavioral health research. The study was conducted to obtain the perspectives of both providers and patients on MAT during pregnancy. The patient data will be reported in a separate manuscript in a future manuscript. This provider pilot study will highlight challenges and opportunities to inform future larger research studies.

\section{Methods}

This qualitative descriptive study elicited in-depth information through semi-structured interviews with healthcare providers. Twelve interviews were conducted by telephone and face-to-face meetings. Eligible providers included those with experience in maternal healthcare, obstetrics, perinatal mental health, addiction, clinical research, and MAT. Overall, six individual telephone interviews, one face-to-face interview and two group interviews (2-3 providers/group) were conducted. Each interview lasted approximately 60 minutes. Permission to audio record was obtained and a facilitator was assigned to recording, time-keeping, and note-taking. Participants were interviewed from October 2017 to August 2018. A gift card incentive was offered to participants who completed the interviews. All interviews were conducted in English. The study was conducted in collaboration with practitioners from the nonacademic University of BLINDED and the Women's Obstetrics Clinic. The study team developed 16 openended questions (Appendix A). Questions included practice characteristics and demographics (age, education, years of practice, setting/patient population served). Interview questions were reviewed for 
relevance and clarity by our partners. Two content area experts modified questions to include practicerelevant probes.

\section{Recruitment}

During the planning phase, the team contacted clinicians and administrators within the medical network systems who served pregnant women with substance use histories to generate a pool of providers through purposive sampling. Providers were recruited by word-of-mouth informal inquiries, email, telephone calls, and through referrals from those who had completed interviews. Participants received study-related information/questions to encourage reflection before interviews and to facilitate discussions. Informed consent was received from all participants. The study reached saturation at $N=$ 12 , with the depth, similarities, and sufficiency of the information gathered from providers.

\section{Sample}

The study sample ( $n=12$ ) consisted of a psychiatrist (1), obstetrician/medical director (1), neonatologist (1), psychologists (3), social workers (3), midwives/nurse practitioners (2), and a mental health nurse practitioner (1), who practiced in outpatient clinic/hospital settings. Approximately half of the providers were also engaged in teaching at the University of BLINDED. Social workers provided services to pregnant women and families with substance use histories in both outpatient and hospital settings. The psychologists and two social workers had backgrounds in addiction and behavioral health counseling as well as with the Child/Welfare system. Provider professional experiences ranged from 3-28 years (Table 1). 


\begin{tabular}{|c|c|}
\hline Sample Characteristics & $\begin{array}{l}\text { Values }(\%) \\
(N=12)\end{array}$ \\
\hline \multicolumn{2}{|l|}{ Age (years) } \\
\hline $20-29$ & $1(8 \%)$ \\
\hline 30 or over & $11(92 \%)$ \\
\hline \multicolumn{2}{|l|}{ Educational level } \\
\hline Bachelors & $0(0 \%)$ \\
\hline Masters & $4(33 \%)$ \\
\hline Doctorate & $8(67 \%)$ \\
\hline \multicolumn{2}{|l|}{ Race } \\
\hline White & $10(83.4 \%)$ \\
\hline Black & $1(8.3 \%)$ \\
\hline Unknown & $1(8.3 \%)$ \\
\hline \multicolumn{2}{|l|}{ Ethnicity } \\
\hline Hispanic & $0(0 \%)$ \\
\hline Non-Hispanic & $12(100 \%)$ \\
\hline \multicolumn{2}{|l|}{ Practice Setting } \\
\hline Inpatient/Outpatient & $5(42 \%)$ \\
\hline Outpatient Clinics (only) & $7(58 \%)$ \\
\hline \multicolumn{2}{|l|}{ Years of Practice } \\
\hline Less than 10 years & $3(25 \%)$ \\
\hline $10-20$ years & $5(42 \%)$ \\
\hline More than 20 years & $4(33 \%)$ \\
\hline
\end{tabular}

Table 1: Sample Characteristics

Five providers (a psychiatrist, nurse practitioners and the obstetrician/medical director) obtained MAT training/waivers through SAMHSA, but the only active prescriber during the study was the psychiatrist. Although waivers were recently obtained, it is unclear why the remaining providers were not prescribing MAT. It is possible that the opportunity for patient induction may not have presented itself, or the referral process to the waivered psychiatrist for prescriptions and behavioral health counseling remained a convenient option for both providers and patients. The psychiatrist had a long history of prescribing MAT over many years in both outpatient and inpatient settings. 


\section{Analysis}

All interview responses were transcribed and analyzed according to an inductive grounded theory

approach. ${ }^{22,23}$ At least three coders from the research team analyzed transcripts and coded each transcript no less than two times, using Microsoft Word/Excel (Microsoft Corporation, Redmond, WA, USA) to initiate coding and NVIVO 12 software (QSR International, Victoria, Australia) in the final analysis. Data were organized by coding schemes and themes. Responses were analyzed by domain and processrelated broad categories were aligned with the inductive nature of qualitative research. ${ }^{22,23}$ Multiple-tiered processes were conducted to assign and reassign codes, inclusive of inputs from three reviewers, until a consensus was built around overarching themes. The study was approved by the University of BLINDED.

\section{Results}

The study borrowed from Anderson's framework for healthcare access ${ }^{24,25}$ and focused on facilitating inhibitory and operational factors that informed the interview questions depicted in the following four domains (Figure 1): Provider perceptions of MAT; Treatment delivery; Access to resources; and Challenges/barriers. Recommendations from providers are reported in a separate section.

\section{Provider Perceptions of MAT, Treatment delivery and Access to resources}

Five central themes (Figure 1) emerged from the three domains (Perceptions of MAT, Treatment delivery, and Access to resources) and are presented in this section.

\section{MAT is a better option for pregnant women with OUD}

Mostproviders $(n=11)$ perceived MAT to be a better option for treatment compared to reports of immediate cessation of drug use recommended by some clinicians during pregnancy. Several providers shared the perceptions of MAT for pregnant women:

"MAT makes so much sense, and for most of them [women], it is a lifeline. It is how they survive. I think without it, they would be back to where they started" (Participant (P) 2, P8).

"Treatment with medications reduces risky behaviors, infections, and other negative factors that compromises the health of pregnant women" (P3).

Many providers $(n=6)$ argued that a safer and controlled environment, as well as well-established current neonatal abstinence syndrome (NAS) treatment modalities, reduced the severity of withdrawal symptoms. In one provider's word, "withdrawal [for babies] outside of the mother is much better than withdrawal inside the mother" (P4). 
Overall, many providers $(n=6)$ with outpatient/inpatient experience, favored buprenorphine, over methadone. Two providers shared the following statement:

"Buprenorphine was more likely to lead to successfully being able to stop use. The majority [patients], find it helpful" (P7).

"People in general on buprenorphine feel the effects of the medication less than methadone" (P5). Most providers $(n=11)$ acknowledged buprenorphine was easily accessible on the streets and the likely source was from prescriptions diverted by patients in treatment.A clinic provider reported that about half of the patients on buprenorphine knew of the benefits during pregnancy and were "already getting it on the street, to manage their addiction as best as they can" (P5), prior to being started on MAT in the clinic.

\section{Pregnancy: A time to promote MAT}

This perception was widely shared by most providers $(n=10)$ who found pregnancy to be a motivating time for change. "Younger women who had not previously sought help for their addiction were motivated to get healthy and found in pregnancy something they want to address" (P5). Providers reported that patients believed MAT would help their babies and prevent withdrawal symptoms. One provider spoke about the potential for behavior change during this period:

"Pregnancy offered a precious opportunity to start fresh. A time when patients were the most motivated" (P7).

\section{Integrated and co-located models can improve treatment}

Recent and ongoing discussions about treatment modalities have shed light on the variability of treatment for pregnant women with OUD. ${ }^{32}$ Providers operated in practices that were linked to referral services such as medical, addictions and psychiatry specialties, as well as to programs in facilities that were separated but proximal to essential clinics throughout the university networks. Most providers ( $n=$ 10) were unclear about their practice delivery models, which were described, as "traditional collaborative," "integrated," "co-located," "embedded," or "traditional substance abuse programs."

Providers $(n=10)$, uniformly described the co-located (or integrated) or "embedded" model as crucial to treatment and viewed the approach more positively than other models. To study providers, practice delivery models were not fully integrated. One outpatient provider commented that the integrated model was, "an effective strategy, not $100 \%$ co-located care, but as close as we have" (P5). The perception of most providers was that it was not enough to have a co-located model, and the most desirable approach was one that was also patient-centered.

\section{Hospitalization and prenatal care link women to MAT and healthcare services}


Hospital admissions provided an opportunity to treat at-risk women who may otherwise be lost to the healthcare system.Providers $(n=8)$ reported an opportunity to "catch them..." (P2) during inpatient admissions when women presented for preterm labor, withdrawal symptoms, or other conditions. Two inpatient providers commented:

"We [hospitals] are their entry into getting care. It's the first time they were getting care, for some women (P1). They [women] are referred appropriately and not discharged without an outpatient plan to continue treatment" (P6).

Outpatient clinic providers also described prenatal visits as a "gateway" (P2) for pregnant women to access healthcare and meet prenatal objectives of engaging women during pregnancy:

"I am like the first step in their process to connect with treatment for women seeking prenatal care and who have typically been struggling with substance abuse for a long time" (P2).

\section{Supportive services improve MAT delivery}

Allproviders $(n=12)$ acknowledged the critical role of social workers in supporting MAT delivery initiated by waivered prescribers. One provider spoke about the work of social workers:

"In our clinic, it's the social worker. We pretty much, the two of us, deal with the bulk of them. They [social workers] are critical for continuity of care. If we didn't have a social worker, we couldn't do any of this. We will work with a social worker to get them into an outpatient facility" (P3, P6).

Social workers described engagement opportunities with the healthcare team beyond prenatal appointments, such as, screening and assessing new/returning pregnant women, coordinating referrals, locating resources, providing psychosocial support, building relationships, and working closely with local/state agencies to identify suitable programs and services.

All providers $n=12$ ) spoke about the diminishing support women with OUD on maintenance MAT received post-pregnancy. Providers described postpartum support as a critical time when women were already overwhelmed with the care of newborns and were therefore at risk of relapsing.

"Women worried about managing their recovery in the context of parenting. Relapse is about two issues: removing the motivator of doing something for their babies... and visitation with providers that occurred

weekly in the third trimester... I worry about the moms once the baby is here and the attention goes

away...our ability to help in the postpartum period...is particularly worrisome" (P7).

Another provider concluded, "You don't stop being an important person when you are not pregnant anymore" (P1).

\section{Challenges and barriers}


Overall four themes emerged in relation to provider/health systems (2) and patient factors (2), when providers were asked about challenges and barriers to MAT delivery (Figure 1).

\section{Lack of provider knowledge, inadequate training, and inconsistent treatment guidelines}

Most providers $(n=8)$ found inpatient medical residents lacked knowledge of current MAT information and updated guidelines for clinical decision making, often "citing old research" (P9). Inpatient providers ( $n$

$=4$ ), reported feeling inadequately prepared to screen and assess the increasing number of pregnant women with OUD on admissions and did not "necessarily feel comfortable" (P7, P9) counseling women without adequate MAT training. Treatment guidelines were reported to be inadequate or absent for postoperative cesarean sections and immediate postpartum because of unresolved MAT concerns. One provider added:

"We don't have written protocols in place. It is an issue when the recommendations can change. Tell us what to do! We want to do the right thing. We want to treat the pain, but not in a way that risks relapse. But then there can be contradictory recommendations given to us" (P7).

\section{Lack of follow-up and inadequate coordination}

For women on MAT maintenance, inpatient providers $(n=5)$ complained that there was "no good plan for follow up and coordination" (P5) with prescribing practitioners whose patients were on MAT. Attempts to communicate with these practitioners during inpatient admissions to determine the right dosage was time-consuming and frustrating when patients did not have prescriber contact information. Similar frustrations were also expressed by outpatient providers $(n=5)$ by the following comments:

"A lot of them [providers] are very busy, so getting someone to call back and confirm their dose can take too long. A provider would have to seek out notes from other providers, to confirm medication dosages" (P7, P12).

A few providers $(n=3)$ found methadone clinics were less forthcoming with information-sharing and one provider added that the clinics "lacked real coordination with goals for treatment."

\section{Lack of external support/resources}

Among the challenges and barriers, providers identified two main themes related to patient factors which impeded treatment. The first factor is the lack of external and additional support and resources for pregnant women with OUD.Most providers $(n=8)$ stated MAT delivery was impacted by limited childcare, transportation, and housing. Providers called transportation a major barrier that affected appointments, work, attendance, timeliness, child visitation/child custody and therefore, treatment. 
"We don't have a good way of addressing a lot of other psychosocial needs they have like helping with transportation" (P5).

"I think we forget sometimes how hard it can be to get across town if you don't have a car. If you're trying to bring along a toddler, or you need to go check in with your probation officer or have to provide a document you can't produce.... Every time I hear about some of the things our clients deal with, I'm amazed they're ever there" (P7).

Providers $(n=9)$ complained about lack of access to residential placement facilities and programs for MAT patients. Few programs existed, particularly for pregnant women with children, and those with a critical need such as the homeless or women living with another drug user. Social workers who coordinate MAT delivery summed up their efforts to locate residential services as beleaguered by:

"Availability, geography, insurance, and transportation... Barriers related to availability of services are the most frustrating... Because when I have a woman who's willing and able and covered, to not be able to place her in treatment is very frustrating" (P2, P12).

A few providers familiar with the justice system $(n=3)$, expressed concerns about MAT access and the criminal justice system. They reported a system that lacked clear guidelines and avenues to communicate with providers and women about MAT resulting in inadequate or no access to MAT for incarcerated pregnant women as reported in the following comment by one provider:

"The courts don't understand when they [women] are on their medication, what the levels are, and might make judgements about the appropriate dose rather than talk to the individual or doctor" (P8).

\section{Stigma, shame, and guilt}

The second major patient factor revealed the theme of stigma, shame, and guilt which providers considered to be barriers to effective MAT delivery. Providers $(n=5)$ reported that women often felt stigmatized by healthcare professionals. Stigma was a barrier to treatment and women were fearful of being discovered to be utilizing MAT and may be the biggest barrier as noted in the following provider comments:

"For the people I see who are on it [MAT], to take care of their kid, but they don't want to have to be on it and they don't want their children to know they are on it. I have one client whose sisters are on treatment; methadone and she goes over to watch the kids in the car when they go to pick up the medications. They feel very stigmatized of being on it [MAT] and needing it" (P3).

"For women there is the stigma of being seen in a drug treatment program, which a major hurdle and discouraged women from keeping appointments. Women were fearful of being discovered to be on MAT" (P1, P11).

"There is also the fear for example, if they tell other doctors or a pediatrician that they're on it, that they might indicate they can't take care of their kids, when they are aware they need to be on it in order to not use. There is a lot of stigma, especially for the people who use it to addiction and pain" (P3). 
Providers revealed women reported feeling ashamed and concerned about negative words from healthcare providers and expressed mixed feelings about staying on MAT. One provider reported, "I hear nurses all the time say it [MAT] is replacing substance for another" (P9), although MAT was prescribed and prescribed by a provider. In another area, post-delivery providers $(n=6)$ stated that their patients expressed guilt about their babies and NAS. One provider shared this view:

"Part of it is guilt. They don't want to see the baby shaking and going through withdrawal. I think probably the biggest thing that prevents them besides the mechanisms of addiction, is the shame and guilt and worry about what's going to happen to their kids" (P8).

\section{Recommendations}

Overall, providers recommended four areas for improving MAT delivery and focused on recommendations to improve both provider barriers/challenges, as well as, patient factors that impeded treatment progress.

\section{Improve patient access to resources and education}

Most providers $(n=10)$, recommended improvements to psychosocial needs to complete MAT delivery. Availability and access to patient resources such as transportation, housing, money, support groups, parenting education and, outreach workers/recovery support personnel impacted comprehensive treatment: "Lack of safe and stable housing impacts the ability to keep custody of their children, a major stressor for women" (P8). A provider with multiple years working with pregnant women found psychosocial needs to be one of the most important factors for effective treatment, and spoke about $\mathrm{d}$ the importance of available resources:

"Just having more resources, more outreach, some peer recovery navigators, and having more access to more residential programs will improve treatment delivery. A peer-to-peer, like another mom who's gone through it and is on the other side, who is in recovery and who was able to keep her kids, I think that would probably be the best help" (P5).

Another factor presented by providers $(n=8)$ was, access to no-cost/free educational materials from government agencies to reinforce messages about OUD, NAS and postpartum conditions. Additionally, child welfare providers, recommended anticipatory guidance teaching, "transparency and working with the parents beforehand" (P1) to reduce fears/anxieties, provide clear guidelines/expectations and prevent child removal/custody battles.

\section{Advance an integrated "one-stop shop" model}

Providers $(n=8)$ recommended an integrated team-based, and a patient-centered approach as the ideal model for pregnant women. Existing evidence-based integrative models appear to be effective. For 
women with OUD, having related services in one place as a "one-stop shop" facilitates collaboration, coordination, and seamless transitions as noted by inpatient providers:

"To have a model where the treatment is done within the OB clinic, would be simple and they might be more likely to follow through [with treatment] if they go to one place. For some women, there's the stigma of being seen in drug treatment program that bothers them. For some, it's just convenience, (or, they are familiar with their OB [obstetrics]), so they want to stay" (P5, P11).

\section{Provide education and training to enhance OUD management}

All providers recommended education and training to lessen provider discomfort with prescribing MAT to pregnant women. Training will reduce knowledge gaps in MAT management of pregnant women and should be embedded into a core curriculum in medical education. Providers $(n=7)$ reported that mentorship training with experienced MAT prescribers, such as psychiatrists, will be beneficial to prenatal providers.

"Colleagues would be more willing to obtain the waiver if they had the opportunity to practice. If I were to start in a position where I was expected to prescribe buprenorphine, I would want to spend a little time in the programs where they already do that. Just to make sure I understand all the subtleties" (P7).

\section{Reduce stigma and promote patient-centeredness}

Providers $(n=7)$ should be made aware of the similarities in addiction and chronic disease management during encounters and exercise more tolerance towards persons with addictions. Substance use disorders may lead to "chronic relapsing, just like diabetes and hypertension" (P11).

"No more insulin for you, is not an option offered to diabetics, after eating sweets and reaching high blood sugar levels" (P11).

Women should therefore not be punished "over a weekend of using," at a time when the need for continued treatment is greatest. Providers should use appropriate terminology and language such as "persons with drug addiction" instead of, "a drug addict," (P6), to reduce stigma and promote patientcentered approaches to advance patient/provider relationships as noted by one provider:

"I think the focus on the relationships is really critical, to addressing the roots of addiction, experiences of childhood trauma or addicted parents, and patients who did not grow up with a lot of support. In keeping people engaged in recovery and utilizing all the services effectively, I would say relationships are the most important part" (P5, P6).

\section{Discussion}


In this qualitative pilot study, we found that concerns about inconsistent practice guidelines and outdated information for clinical decision-making persisted among providers, despite attempts to close known training gaps in MAT delivery. ${ }^{18}$ This study identified significant potential areas for focused training that fulfills SAMHSA's recommendation for healthcare provider education and assistance. ${ }^{18}$ For example, providers perceived that access to practical hands-on training opportunities or an embedded program with experienced MAT psychiatrists, will prepare clinicians, such as obstetricians, to prescribe and better manage patients with OUD. We think that this approach will meet the specific needs of practitioners and enhance the Provider Clinical Support System mentorship program. ${ }^{18}$

A less stringent definition of MAT "qualifying practitioners," afforded through the "Support for Patients and Communities Act,"' can potentially create a wider pool of trained professionals to bridge training gaps. ${ }^{26}$ The new cadre of waivered practitioners, including, "Clinical Nurse Specialists, Certified Registered Nurse Anesthetists and Certified Nurse Midwifes,"' will improve access to more and timely providers who can address barriers such as lack of MAT continuation among incarcerated pregnant women reported. ${ }^{27}$

This study emphasizes opportunities for MAT initiation during inpatient pregnancy-related admissions to improve healthcare access for women who may otherwise delay entry fearing stigmatization and criminalization, including incarceration. ${ }^{6}$ Sakala and Corry ${ }^{28}$ reported almost all childbirth occur in hospitals; therefore, inpatient providers can begin MAT induction during admission for untreated patients with OUD, where indicated. Inpatient care is also shaped by multidisciplinary approaches that can deploy pathways to early identification, referrals, coordinated care, and linkages to community agencies for substance-dependent pregnant women to improve care coordination. ${ }^{18}$ Provider awareness of major access points increases optimal maternal and neonatal health outcomes for reproductive age women who are likely to have unintended pregnancies and find themselves pregnant while using. ${ }^{29}$ Access to women at risk, may be lost if providers fail to recognize the window of opportunity presented in pregnancy.

Wright et al. ${ }^{30}$ recommended screening, brief interventions, referrals, and treatment (SBIRT) in outpatient settings to identify pregnant women for addiction management. In this study, providers also found that continuing the trajectory of outpatient accessibility through clinics is critical for MAT delivery. Traditional outpatient prenatal settings having been wholly established as major access points for early maternal interventions/services face multiple challenges to integrate and coordinate addiction services, mostly provided through specialty treatment programs. ${ }^{31}$ We believe that the first step is for providers to recognize the practice settings as a significant venue for delivering MAT to pregnant women.

Although integrated prenatal and addiction services are reportedly effective, ${ }^{30}$ office-based opioid treatment, opioid treatment programs, hub and spoke and other practice models, underscore the complexity of MAT delivery. ${ }^{32}$ An effective integrated model, often referred to as co-located, bears the hallmarks of accessibility, coordination and communication. ${ }^{32}$ In this study, we found provider practices were not truly co-located or integrated since referrals for addictions or prenatal care were located 
separately from the primary site. Lack of clarity about practice models is not uncommon among providers, the reasons for which are beyond the scope of this study. ${ }^{32}$ Interventions for integrated "onestop shop" models, as proposed in this study, have shown notable success in coordinated care, particularly among non-pregnant HIV populations. ${ }^{32,33}$ Implementing such a program will require realigning reimbursements from current sole/primary provider and fee-for-service payer systems (by individual procedure/visit) to innovative proposals, under consideration, such as value-based bundled maternity payments or episodic payments for billed services. ${ }^{34}$ The tenements of value-based care, quality, coordination of services, interdisciplinary approach, and patient-centered care parallels the integrated model proposed in this study. The feasibility of the integrated model needs further evaluation particularly in low MAT provider access areas.

Overall, providers perceived MAT as a promising modality to treatment, consistent with previous studies that indicated a preference for buprenorphine over methadone during pregnancy. ${ }^{12}$ For critical barriers to MAT delivery, such as lack of transportation and residential housing to meet the specific needs of pregnant women (i.e. mothers with small children and pregnant women victimized in domestic violence situations), funding support remains largely insufficient and will likely continue to impede comprehensive treatment. ${ }^{6,30,35}$ Non-traditional and innovative funding streams can be tapped to assist low-income women. ${ }^{35}$ Provider conclusions of MAT "as a lifeline" will be explored in future studies that focus on patient perspectives.

Stigma of addiction has been well documented in the literature. ${ }^{30,35,36}$ In this study, providers called for interventions such as a cultural shift in thinking about addictions as a chronic disease. A patient-centered approach based on tolerance towards persons with addictions can decrease stigmatization and improve treatment. ${ }^{37}$ Brief interventions during initial encounters utilizing a patient-centered approach such as motivational interviewing can also be effective. ${ }^{30}$

Through provider input, this study reveals vulnerabilities of deficient support that could lead to relapse in postpartum women. $6,31,35$ Although Medicaid offers postpartum follow-up through 60 days, researchers/clinicians agree that the service period is inadequate to meet recovery goals. ${ }^{31,35}$ Under the Affordable Care Act, postpartum coverage that includes addiction and mental health services can be expanded to a full year, which 36 states and the District of Columbia have adopted. ${ }^{38}$ More evidence is needed to determine the impact on maternal morbidity and mortality, though some studies show that supportive postpartum resources increase treatment adherence, reduce relapse rates and hospitalizations, and improve bonding, breastfeeding and paternal involvement. ${ }^{31}$ Literature from supportive peer recovery coach studies are promising, but there is little evidence emerging among pregnant and postpartum women. ${ }^{39}$

\section{Conclusions}


Overall, providers want better coordination of services across disciplines, enhanced training and treatment readiness, access to qualified prescribing providers, and supportive psychosocial and educational resources for better management of pregnant women with OUD. Utilizing a team-based integrated or "one-stop shop" approach, where MAT and prenatal care are delivered simultaneously, has possibilities for an effective delivery model that should be further explored. The study is limited by a small sample of providers selected from urban outpatient/inpatient practices located within an academic network, which may lack generalizability to other settings, particularly to rural areas. We expect to utilize the findings from this pilot study to inform larger and future studies and innovative models. The strength of the study comes from sharing the perspectives of MAT delivery from experienced providers who have had direct interactions with pregnant women with OUD. Healthcare providers have a window of opportunity to improve MAT delivery at a time when the woman is more motivated to change and to utilize health services to a greater extent. Healthcare providers can lead the pathway to improved care for pregnant women with OUD. Such a strategy has long-term implications for the recovery process, relapse prevention, and health outcome improvements for pregnant and reproductive age women with substance use disorders.

\section{List Of Abbreviations}

DATA - Drug Abuse Treatment Act

MAT - Medication-assisted treatment

NAS - Neonatal abstinence syndrome

OUD - Opioid use disorder

SAMHSA - Substance Abuse and Mental Health Services Administration

SBIRT - Screening, brief interventions, referrals, and treatment

\section{Declarations}

\section{Ethics approval and consent to participate}

The authors sought approval was sought from the University of Maryland Institutional Review Board

\section{Consent for publication}

Not applicable

\section{Availability of data and materials}


The data collected and analyzed during the current study are not publicly available due to patient privacy but are available from the corresponding author on reasonable request.

\section{Competing interests}

The authors declare that they have no competing interests.

\section{Funding}

This study was supported by the Parent Project Grant \#10020715, Patient-Centered Involvement in Evaluating the Effectiveness of Treatments (PATIENT) and Patient-Centered Outcomes Research (PCOR) projects. Parent Project PI: C. Daniel Mullins, PhD.

Parent Project Funding Source: Agency for Healthcare Research and Quality, US Department of Health and Human Services, 5600 Fishers Lane, Rockville, MD 20857. www.ahrq.gov

The authors declare that no funding body had a role to play in the design of the study, the collection, analysis, and interpretation of data, nor in the writing of the manuscript.

\section{Authors' contributions}

DTG designed, implemented, collected, analyzed data, recorded results, and developed the manuscript; CW contributed to patient recruitment and draft reviews and offered feedback to improve the entire manuscript; RV and DMQ coded and interpreted data; SS developed tables and schematics, and, contributed to the results section. LEG was a major contributor to the initial writing of the manuscript and reviewed the preliminary draft; and, FTS also contributed to the writing of the manuscript, offered feedback, monitored progress, and acted as timekeeper. All authors reviewed and approved the final manuscript.

\section{Acknowledgements}

Claudia E. Nettey MPH, BDS of the University of Maryland School of Nursing contributed to manuscript preparation and provided editing assistance.

\section{References}

1. Haight SC, Ko JY, Tong VT, Bohm MK, Callaghan WM. Opioid use disorder documented at delivery hospitalization - United States, 1999-2014. MMWR Morb Mortal Wkly Rep. 2018;67(31):845-9. http://dx.doi.org/10.15585/mmwr.mm6731a1. 
2. Dowell D, Haegerich TM, Chou R. CDC guideline for prescribing opioids for chronic pain--United States. JAMA. 2016;315(15)1624-45. doi:10.1001/jama.2016.1464

3. Paulozzi LJ, Mack KA, Hockenberry JM. Vital signs: variation among states in prescribing of opioid pain relievers and benzodiazepines - United States, 2012. Division of Unintentional Injury Prevention, National Center for Injury Prevention and Control, CDC. MMWR Morb Mortal Wkly Rep. 2014;63(26):563-8. https://www.ncbi.nlm.nih.gov/pubmed/24990489.

4. Martin CE, Longinaker N, Terplan M. Recent trends in treatment admissions for prescription opioid abuse during pregnancy. J. Subst. Abuse Treat. 2014;48(1):37-42.

5. American Psychiatric Association. Diagnostic and Statistical Manual of Mental Disorders: DSM-5. 5th ed. Arlington, VA; 2013.

6. American College of Obstetricians and Gynecologists Committee on Obstetric Practice \& American Society of Addiction Medicine. Opioid use and opioid use disorder in pregnancy (ACOG Committee Opinion No. 711). https://www.acog.org/-/media/Committee-Opinions/Committee-on-ObstetricPractice/co711.pdf?dmc=1\&ts=20180803T1619512856. Accessed 30 Apr 2019

7. Behnke M, Smith VC, Committee on Substance Abuse, Committee on Fetus and Newborn. Prenatal substance abuse: short- and long-term effects on the exposed fetus. Pediatrics. 2013;131(3):e10091024. doi:10.1542/peds.2012-3931.

8. Maryland Department of Health and Mental Hygiene Prevention and Health Promotion Administration. Maryland Maternal Mortality Review 2016 Annual Report: Health - General Article $\S$ 13-1203-1207. 2016. http://healthymaryland.org/wpcontent/uploads/2011/05/MMR_Report_2016_clean-copy_FINAL.pdf. Accessed Apr 302019.

9. Virginia Department of Health. Pregnancy-associated deaths from drug overdose in Virginia, 19992007: a report from the Virginia Maternal Mortality Review Team. Richmond, VA, 2015. http://www.vdh.virginia.gov/content/uploads/sites/18/2016/04/Final-Pregnancy-AssociatedDeaths-Due-to-Drug-Overdose.pdf. Accessed Apr 302019.

10. Patrick SW, Davis MM, Lehmann CU, Cooper WO. Increasing incidence and geographic distribution of neonatal abstinence syndrome: United States 2009 to 2012. J Perinatol. 2015;35:650-655. doi:10.1038/jp.2015.36.

11. Substance Abuse and Mental Health Services Administration. Medication-assisted treatment for opioid addiction in opioid treatment programs: Treatment Improvement Protocol (TIP) Series 43. HHS Publication SMA 12-4214, Rockville, MD: Center for Substance Abuse Treatment, 2005. http://store.samhsa.gov/product/TIP-43-Medication-Assisted-Treatment-for-OpioidAddiction-inOpioid-Treatment-Programs/SMA12-4214. Accessed Apr 302019.

12. Jones HE, Finnegan LP, Kaltenbach K. Methadone and buprenorphine for the management of opioid dependence in pregnancy. Drug. 2012;72(6):747-757. doi:10.2165/11632820-000000000-00000

13. Sporer KA. Buprenorphine: A primer for emergency physicians. Ann. Emerg. Med. 2004;43(5):580584. doi: 1016/S0196064403012058 
14. Mattick RP, Breen C, Kimber J, Davoli M. Methadone maintenance therapy versus no opioid replacement therapy for opioid dependence. Cochrane Database Syst, Rev. 2009;(3):CD002209. doi:10.1002/14651858.CD002209.pub2.

15. Mattick RP, Breen C, Kimber J, Davoli M. Buprenorphine maintenance versus placebo or methadone maintenance for opioid dependence. Cochrane Database Syst. Rev. 2014;(2):CD002207. doi:10.1002/14651858.CD002207.pub4.

16. Kampman K, Jarvis M. American Society of Addiction Medicine (ASAM) national practice guideline for the use of medications in the treatment of addiction involving opioid use. J Addict. Med. 2015;9(5):358-367. doi:10.1097/ADM.0000000000000166.

17. Short VL, Hand DJ, MacAfee L, Abatemarco DJ, Terplan M. Trends and disparities in receipt of pharmacotherapy among pregnant women in publicly funded treatment programs for opioid use disorder in the United States. Subst. Abuse Treat. 2018;89:67-74. https://doi.org/10.1016/j.jsat....

18. Substance Abuse and Mental Health Services Administration. A collaborative approach to the treatment of pregnant women with opioid use disorders. HHS Publication No. (SMA) 16-4978, Rockville, MD, 2016. Available online at http://store.samhsa.gov/. Accessed Apr 30

19. Substance Abuse and Mental Health Services Administration. (2018). Clinical guidance for treating pregnant and parenting women with opioid use disorder and their infants. HHS Publication No. (SMA) 18-5054. Rockville, MD: Substance Abuse and Mental Health Services Administration.

20. The Office of the National Drug Control Policy. The President's commission on combating drug addiction and the opioid crisis. Available online at https://www.whitehouse.gov/sites/whitehouse.gov/files/images/Final_Report_Draft_11-152017.pdf. Accessed Apr 302019.

21. Arnaudo CL, Andraka-Christou B, Allgood K. Psychiatric co-morbidities in pregnant women with opioid use disorders: prevalence, impact, and implications for treatment. Addict. Rep. 2017;4(1):1-13.

22. Charmaz K. Constructing grounded theory: a practical guide through qualitative analysis. Sage Publications; 2006.

23. Singh S, Estefan A. Selecting a grounded theory approach for nursing research. Qual. Nurs. Res. 2018; doi:https://doi.org/10.1177/2333393618799571

24. Aday LA, Andersen R. A framework for the study of access to medical care. Health Serv. Res. 1974;9(3):208-220.

25. Babitsch B, Gohl D, Von Lengerke T. Re-visiting Andersen's behavioral model of health services use: A systematic review of studies from 1998-2011. GMS Psychosoc. Med. 2012;9(Doc 11). doi:10.3205/psm000089.

26. H.R.6 - 115 ${ }^{\text {th }}$ Congress (2017-2018). Substance Use-Disorder Prevention That Promotes Opioid Recovery and Treatment for Patients and Communities (Support for Patients and Communities) Act. Public Law 271, 2018. Available online at https://www.congress.gov/bill/115th-Congress/housebill/6/text. Accessed 30 Apr 2019. 
27. Ghidei L, Ramos SZ, Brousseau C, Clarke JG. Prison: Pipeline to women's preventative health. R I Med. J. 2018;23-26. Available online at http://www.rimed.org/rimedicaljournal/2018/10/2018-10-23obgyn-ghidei.pdf. Accessed Apr 30

28. Sakala C, Corry MP. Evidence-based maternity care: What it is and what it can achieve. New York: Milbank Memorial Fund, Available online at http://www.milbank.org/reports/0809/MaternityCare/0809MaternityCare.html. Accessed Apr 30 2019.

29. Heil SH, Jones HE, Arria A, et al. Unintended pregnancy in opioid-abusing women. Subst. Abuse Treat. 2011;40(2):199-202. doi:10.1016/j.jsat.2010.08.011.

30. Wright TE, Terplan M, Ondersma SJ, et al. The role of screening, brief intervention, and referral to treatment in the perinatal period. J. Obstet. Gynecol. 2016;215(5):529-547. doi:10.1016/j.ajog.2016.06.03.

31. Gopman S. Prenatal and postpartum care of women with substance use disorder. Gyn. Clin. N. Am. 2014;41:213-228. doi.org/10.1016/j.ogc.2014.02.004

32. Chou R, Korthuis PT, Weimer M, Bougatsos C, Blazina I, Zakher B, et al. Medication-assisted treatment models of care for opioid use disorder in primary care settings. Agency for Healthcare Research and Quality, Report No.:16(17)-EHC039-EF (Technical Briefs, No. 28), Rockville, MD: Pacific Northwest Evidence-based Practice Center, 2016. Available online at https://www.ncbi.nlm.nih.gov/books/NBK402352/. Accessed Apr 30

33. United States Department of Health and Human Services. Integrating buprenorphine therapy into HIV primary care settings. Rockville, MD: Health Resources and Services Administration, HIV/AIDS Bureau, Special Projects of National Significance Program, 2012. Available online at http://hab.hrsa.gov/abouthab/files/hab_spns_buprenorphine_monograph.pdf. Accessed Apr 30

34. American College of Obstetricians and Gynecologists (Committee on Health Economics and Coding). Value-based payments in obstetrics and gynecology. ACOG Committee Opinion No. 744. 2018; 132; e53-59. Available online at https://www.acog.org/Clinical-Guidance-and-Publications/CommitteeOpinions/Committee-on-Health-Economics-and-Coding/Value-Based-Payments-in-Obstetrics-andGynecology? IsMobileSet=false Accessed Apr 30

35. Howell E, Golden O, Beardslee W. Emerging opportunities for addressing maternal depression under Washington, D.C.: Urban Institute, 2013. Available online at http://ascend.aspeninstitute.org/legacy/resources/412758-Emerging-Opportunities-for-AddressingMaternal-Depression-under-Medicaid.pdf. Accessed Apr 302019.

36. Stone R. Pregnant women and substance use: fear, stigma, and barriers to care. Health Justice. 2015;3(2). doi:10.1186/s40352-015-0015-5.

37. Mullins D, Abdulhalim AM, Lavallee DC. Continuous patient engagement in comparative effectiveness research. JAMA, 2012;307(15):1587-1588.

38. Ranji U, Gomez I, Salganicoff A. Expanding postpartum Medicaid coverage. Washington D.C.: Henry J Kaiser Family Foundation Issue Brief, 2019. 
39. Bassuk E, Hanson J, Greene N, et al. A peer-delivered recovery support for addictions in the United States: A systematic review. J. Subst. Abuse Treat. 2016;63:1-9. doi:10.1016/j.jsat.2016.01.003.

\section{Figures}

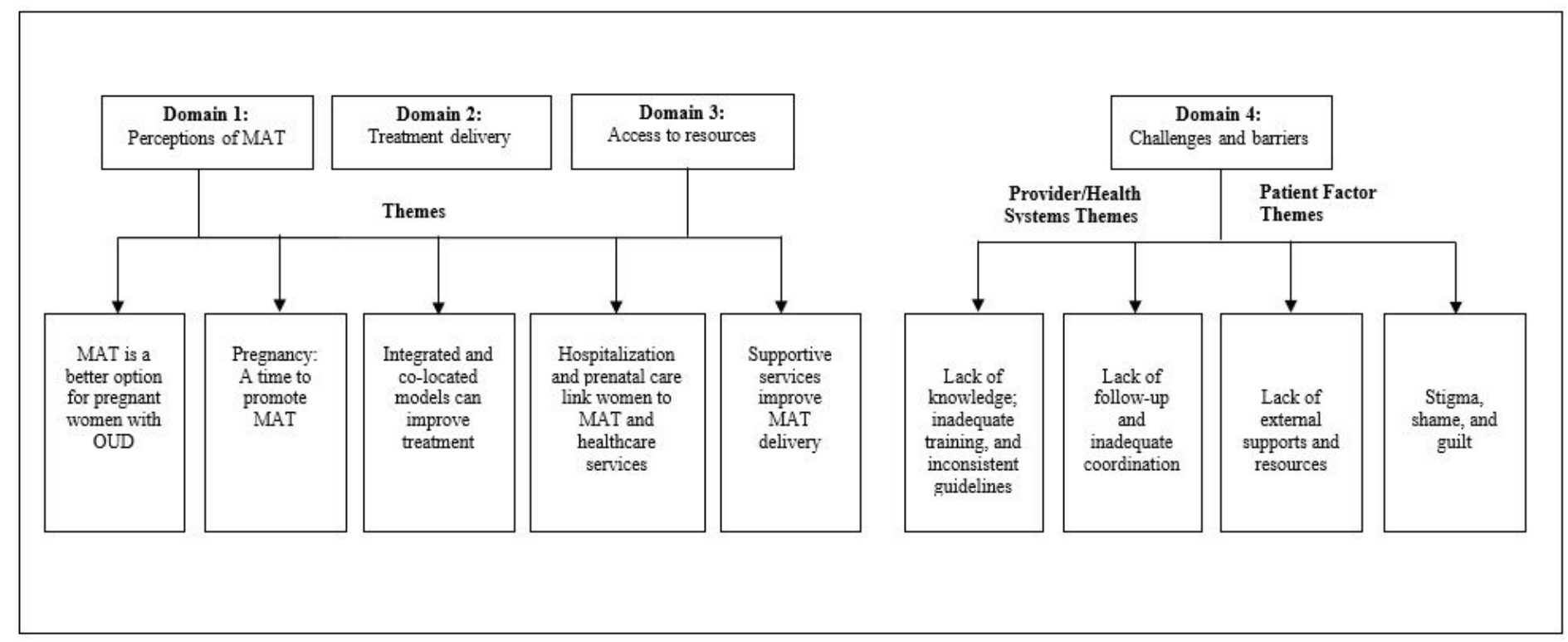

Figure 1

Provider Study Domains and Themes

\section{Supplementary Files}

This is a list of supplementary files associated with this preprint. Click to download.

- AppendixA.docx 